\title{
Scattered Packet Method for the Simulation of the Spatio-temporal Evolution of Local Perturbations
}

\author{
P. GAUBERT ${ }^{\mathrm{a}}$, L. VARANI ${ }^{\mathrm{a}, *}$, J. C. VAISSIÈRE ${ }^{\mathrm{a}}$, J. P. NOUGIER ${ }^{\mathrm{a}}$, E. STARIKOV ${ }^{\mathrm{a}, \mathrm{b}}$, \\ P. SHIKTOROV ${ }^{b}$ and V. GRUZHINSKIS ${ }^{b}$

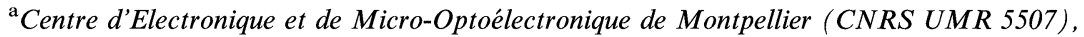 \\ Université Montpellier II, Place Eugène Bataillon, 34095 Montpellier cedex 5, France; \\ ${ }^{\mathrm{b}}$ Semiconductor Physics Institute, Goshtauto 11, 2600 Vilnius, Lithuania
}

\begin{abstract}
To calculate the noise in submicron structures we need the knowledge of the local noise sources and the generalised impedance fields. The scattered packet method has been used to obtain both quantities at the same microscopic level. Numerical procedures used for the calculation of velocity and energy noise sources and the simulation of the spatiotemporal evolution of local perturbations introduced on velocity or energy are described and some results obtained for p-type silicon are given.
\end{abstract}

Keywords: Noise; Impedance field; Scattered Packet Method

\section{INTRODUCTION}

The classical impedance field method [1] is one of the most powerful techniques to calculate electronic noise in semiconductor structures. This method fails when applied to deep submicron devices due to the presence of spatial correlations between noise sources [2]. To overcome this drawback, a new technique (generalised impedance field) has been developed in the frame of a hydrodynamic simulator [3]. Analogously to the classical method, the calculation of electronic noise requires the knowledge of two quantities: the local noise source and the generalised impedance fields. Instead of using a hydrodynamic approach to obtain the generalised impedance fields and a Monte Carlo simulation for the noise sources, we have used the Scattered Packet Method [4-6] to compute both quantities at the same microscopic level of description.

\section{NUMERICAL PROCEDURE}

We have developed the Scattered Packet Method (SPM) a few years ago in order to solve the Boltzmann equation for carriers in semiconductors using only the output term in order to increase the stability of the resolution. It follows the spatiotemporal evolution of the carrier population $n$ in different cells of the phase space using an evolution operator $[B]$ that gives $n(t+\Delta t)$ when applied to

*Corresponding author. Tel.: (+33) 467143822, Fax: (+33) 467547134, e-mail: varani@cem2.univ-montp2.fr 
$n(t)$. Written in a matrix form:

$$
[n(t+\Delta t)]=[B][n(t)]
$$

this operator can be represented using three matrices corresponding to the displacement in real space $\left[B_{r}\right]$, displacement in $\mathbf{k}$-space $\left[B_{E}\right]$ and to the collisions $\left[B_{\text {coll }}\right]$ :

$$
[B]=\left[B_{r}\right]+\left[B_{E}\right]+\left[B_{\text {coll }}\right]-2[I]
$$

$[I]$ is the identity matrix.

We choose a time step $\Delta \mathrm{t}$ sufficiently small (between 0.1 and $1 \mathrm{fs}$ ) in order to follow the transient evolution of the Boltzmann equation and to verify that during the time interval a carrier can only go from one cell to its neighbours in $\mathbf{k}$ and $\mathbf{r}$ spaces.

In that case the matrix $\left[B_{r}\right]$ is tridiagonal for $1 \mathrm{D}$ simulations in real space. The matrix elements depend only of the value $\mathbf{k}$ of the wave vector of the initial mesh (we can neglect the acceleration of the electric field) and are calculated using the overlap of the initial cell and the others cells (in r-space) after the time interval $\Delta \mathrm{t}$.

The matrix elements of $\left[B_{E}\right]$ are given by the overlap of the initial cell and its neighbours (between 1 and 9) in $\mathbf{k}$-space after the time interval $\Delta \mathrm{t}$. Values are tabulated for different electric fields.

Using a small sub-mesh we calculate the probability for a carrier to go from one cell to the others cells of the $\mathbf{k}$-space due to impurity scattering and interactions with acoustic and optical phonons. The corresponding matrix $\left[B_{\text {coll }}\right]$ is sparse and tabulated.

Finally, the matrix element $B_{M L}$ of $[B]$ is the transition probability from the cell number $L$ to the cell number $M$ during the time interval $\Delta t$.

In the frame of the generalised impedance field method [7], we have calculated the noise sources and the generalised impedance fields using the SPM.

\section{Noise Sources}

The matrix $\left[B_{\text {coll }}\right]$ is employed to obtain the local noise sources corresponding to the acceleration fluctuations of velocity and energy [8] which are given by:

$$
\tilde{S}_{\dot{\alpha} \dot{\beta}}=\frac{1}{N \Delta t} \sum_{L} N_{L} \sum_{M} B_{M L}\left(\alpha_{M}-\alpha_{L}\right)\left(\beta_{M}-\beta_{L}\right)
$$

$N$ is the total number of carriers used in the simulation, $N_{L}$ the population of the cell number $L, \alpha_{L}, \alpha_{M}, \beta_{L}$ and $\beta_{M}$ are the hydrodynamic velocity $(v)$ and energy $(\varepsilon)$ corresponding to this different cells in the $k$ space. For p-type silicon, results are presented, versus the mean energy (different static electric fields applied to the material) on Figure 1 for velocity (a) and energy (b) and different doping levels between $2.510^{14} \mathrm{~cm}^{-3}$ and $10^{18} \mathrm{~cm}^{-3}$. Full and open
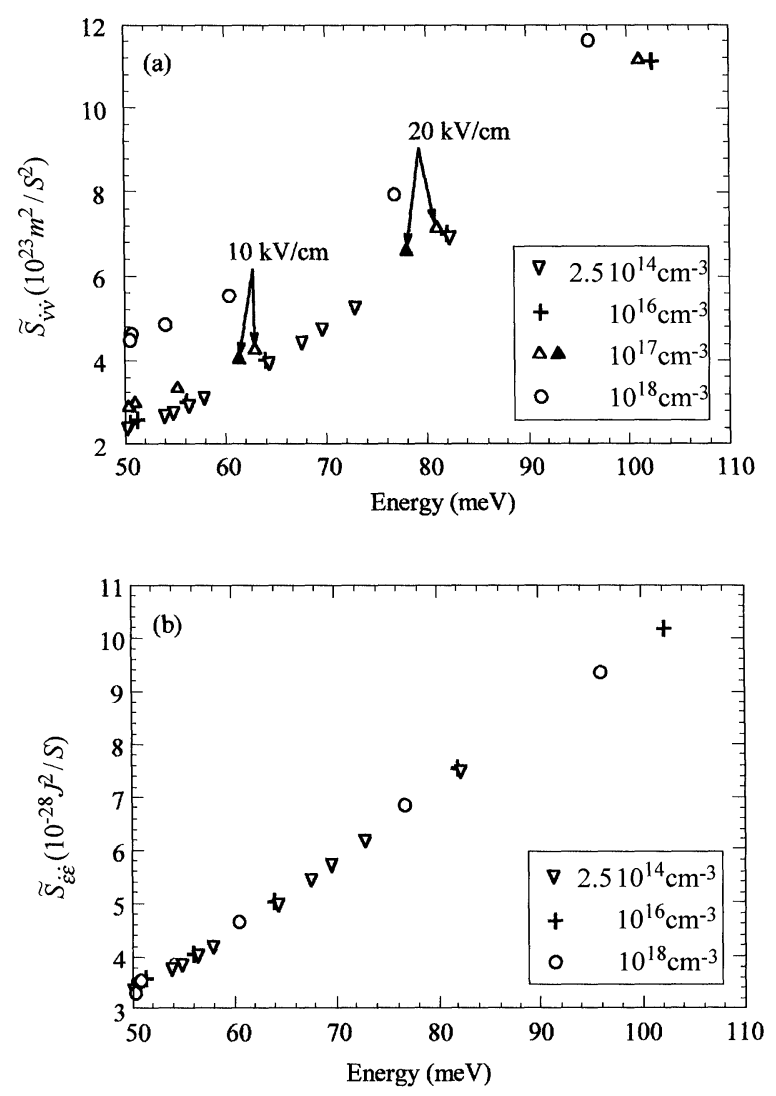

FIGURE 1 Local noise sources versus energy for p-type $\mathrm{Si}$ and different doping levels associated with velocity (a) and energy (b) accelerations. 
triangles on (a) correspond to different sizes used for the k-space mesh in the simulation. The velocity noise source increases with energy and doping concentration while the local noise source associated with the energy shows a quasi-linear behaviour independently of the doping. The crosscorrelated noise source term between velocity and energy decreases, starting from zero, and does not depend on the doping. This source is not shown here because its contribution to the total noise is generally negligible.

\section{Generalised Impedance Fields}

To calculate the generalised impedance field, it is necessary to follow the spatio-temporal evolution of a local perturbation of velocity or energy. Starting from a stationary state, the perturbation is introduced by modifying the local carrier distribution function. A special procedure has been developed in order to perturb only one parameter (for instance velocity) without changing the other (energy). If $N_{S}(k, \theta, x)$ is the stationary population of the cell $(k, \theta)$ in $x$, we transfer a small number $\Delta N$ of carriers from the cell $a$ to the cell $b$ as shown on Figure 2. The perturbation must be small in order to preserve the linearity of the response of the system and obtain the small signal generalised impedance field:

$$
\Delta N=N_{S}(k, \theta, x) F(x) C_{v}
$$

with $C_{v}=\Delta v(x) /\left(2 v_{p}\right) ; v_{p}$ is the absolute value of the sum of the velocities of carriers located at $\mathrm{x}$ with a direction $\theta$, between $x$ and the wave vector $k$, greater than $\pi / 2 ; \Delta v(x)$ is the value of the local perturbation we want to introduce at $x$. Typically $\Delta v(x)=0.1 v(x)$ and $10^{3} \mathrm{~m} / \mathrm{s}$ for low values of $v(x)$. $F(x)$ with $0 \leq F(x) \leq 1$ is a function of $x$ adjusted to obtain a narrow gaussian shape along $x$. Then using the evolution operator coupled to a Poisson solver, we follow the temporal evolution of the perturbation of the distribution function along the electric field $(\theta=0$ and $\theta=\pi)$ presented

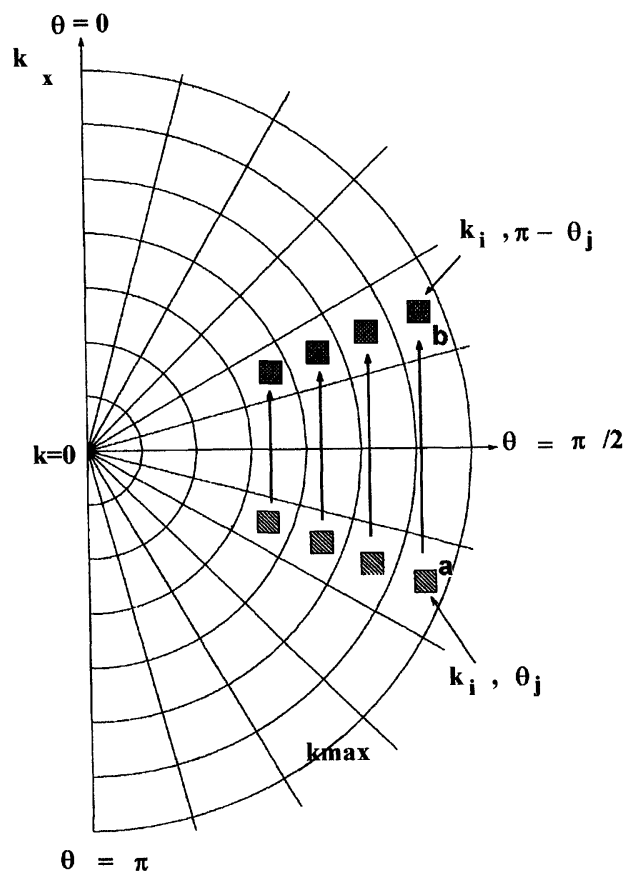

FIGURE 2 Introduction of a local perturbation of the velocity (without perturbing the energy) in the $\mathrm{k}$ space in spherical coordinate at point $\mathrm{x}$.

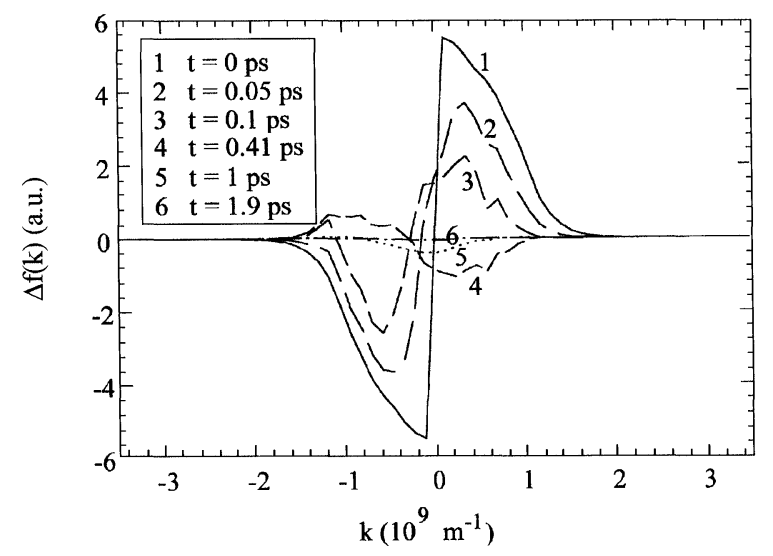

FIGURE 3 Temporal evolution of the perturbation of the distribution function along the field direction at point $x_{0}=$ $0.6 \mu \mathrm{m}$ where the initial perturbation on velocity was introduced.

on Figure 3 for $x_{0}=0.6 \mu \mathrm{m}$ where the initial perturbation (curve 1) of velocity was introduced. Starting from this intitial conditions, the 


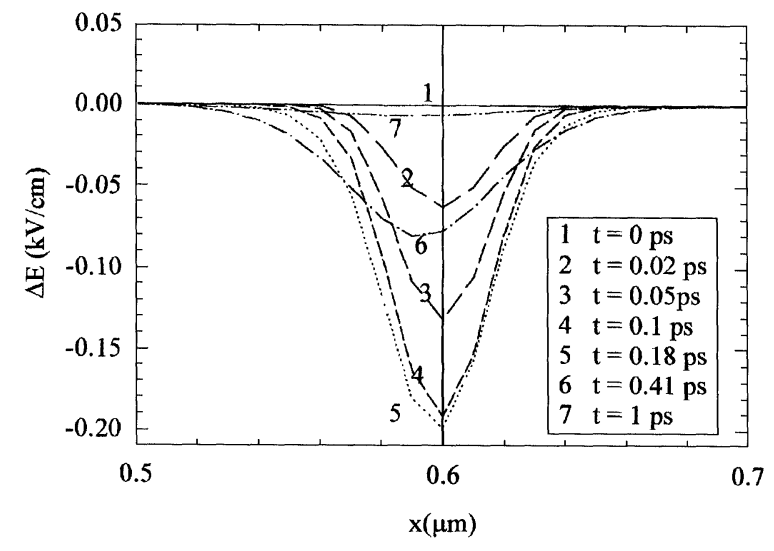

FIGURE 4 Response of electric field for different times when a velocity perturbation is applied at $x_{0}=0.6 \mu \mathrm{m}$ at $t=0 \mathrm{ps}$ in a p-type Si resistance. $N_{A}=10^{17} \mathrm{~cm}^{-3}, E=10 \mathrm{kV} / \mathrm{cm}$.

spatio-temporal evolution of the electric field is calculated and presented in Figure 4 for an applied electric field $E=10 \mathrm{kV} / \mathrm{cm}$. At time $t=0$, we have introduced a small perturbation of the local velocity (and distribution function), but the local energy, carrier concentration $n(x)$ and electric field (see curve 1) are not modified. Then the local electric field is perturbed and a maximum is observed at about $0.2 \mathrm{ps}$. The spatial integration of this perturbation gives the voltage response $G_{U}^{v}\left(x_{0}, t\right)$ at the device contacts plotted on Figure 5

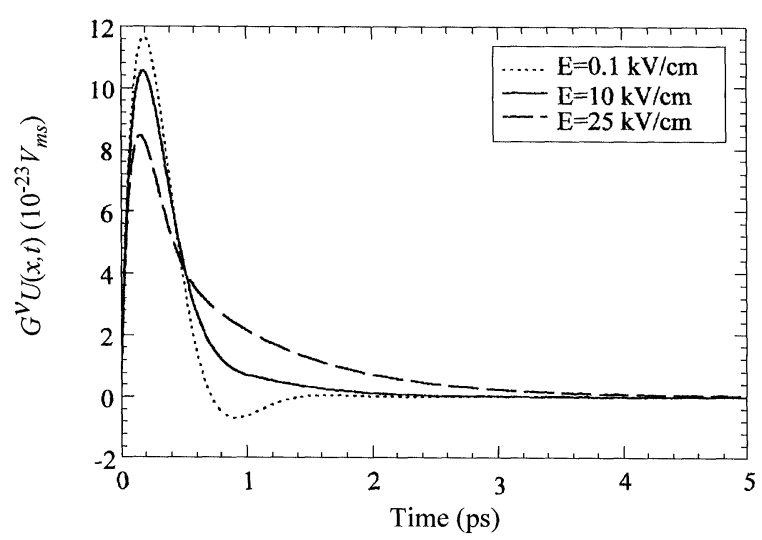

FIGURE 5 Voltage response function for a perturbation of velocity applied at $x_{0}=0.6 \mu \mathrm{m}$ for three electric fields; in a $1 \mu \mathrm{m}$ p-type $S i$ resistance, $N_{A}=10^{17} \mathrm{~cm}^{-3}$. for three different electric fields:

$$
G_{U}^{v}\left(x_{0}, t\right)=\left[U\left(x_{0}, t\right)-U_{S}\right] /\left[\Delta v N_{S}\left(x_{0}\right)\right]
$$

$\Delta v N_{S}\left(x_{0}\right)$ is the total variation of the velocity introduced at $x_{0}$. This sum must be extended to all the cells of the $x$ mesh if the initial perturbation is not exactly located at $x=x_{0}$. By Fourier transforming the response function we calculate the generalised impedance fields associated with velocity. Figure 6 gives the real part, imaginary part and modulus of the generalised impedance field $\nabla Z_{v}$ associated with velocity for three electric fields. A similar procedure can be used [8] to calculate the generalised impedance field associated with energy. In that case the local energy perturbation is introduced by moving in the kspace part of the carriers to higher energy without changing the direction of their velocity. Using this procedure, the local energy is increased and the local velocity $\Delta v(x)$ is modified. To suppress this perturbation of the velocity we use the same procedure as described before to introduce a velocity perturbation without changing the energy. Then the voltage response function and the generalised impedance fields associated with energy are calculated. Using the different noise sources and generalised impedance fields the

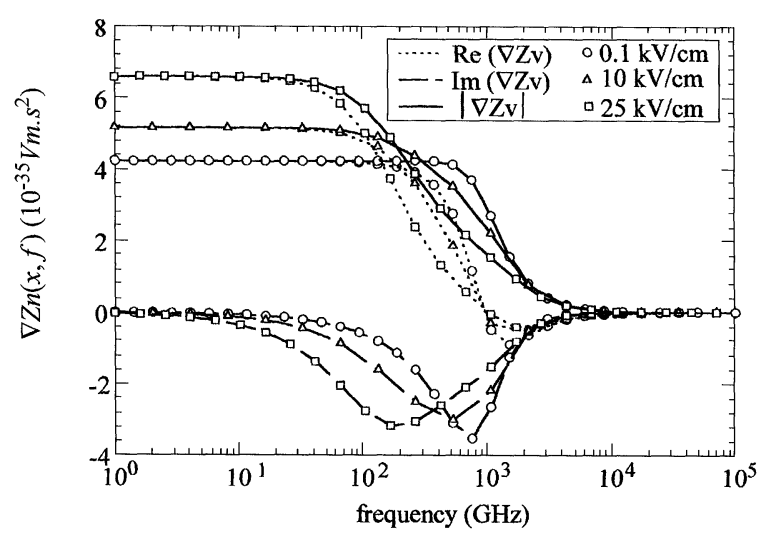

FIGURE 6 Real part, imaginary part and modulus of the generalised impedance field associated with velocity for three electric fields in a $1 \mu \mathrm{m}$ p-type Si resistance, $N_{A}=10^{17} \mathrm{~cm}^{-3}$. 


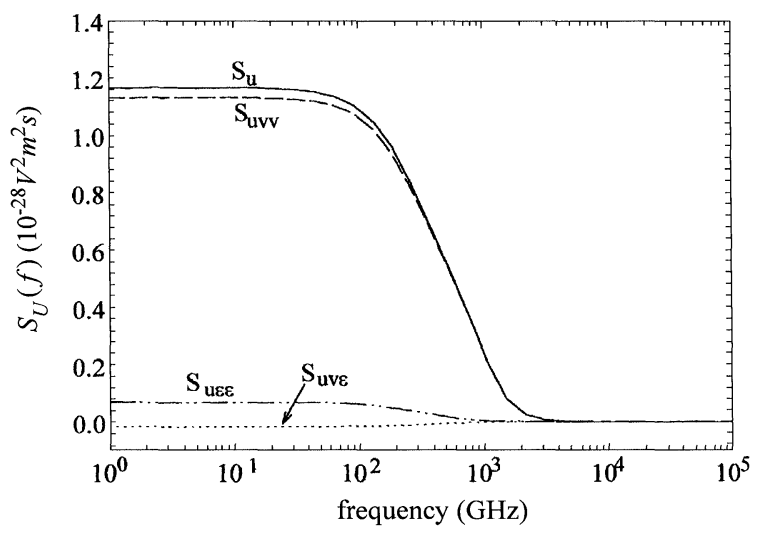

FIGURE 7 Spectral density of voltage fluctuation and its different contributions associated with velocity-velocity $(v v)$, energy-energy $(\varepsilon \varepsilon)$ and velocity-energy $(v \varepsilon)$ terms for a $1 \mu \mathrm{m}$ p-type Si resistance, $N_{A}=10^{17} \mathrm{~cm}^{-3}, E=10 \mathrm{kV} / \mathrm{cm}$.

spectral density of voltage and its different contributions are obtained:

$$
\begin{aligned}
S_{U}(f)= & \int_{0}^{L} \nabla Z_{v}(x, f) \tilde{S}_{\dot{v} \dot{v}}(x) n_{s}(x) \nabla Z_{v}^{*}(x, f) d x \\
& +\int_{0}^{L} \nabla Z_{\varepsilon}(x, f) \tilde{S}_{\dot{\varepsilon} \dot{\varepsilon}}(x) n_{s}(x) \nabla Z_{\varepsilon}^{*}(x, f) d x \\
& +\int_{0}^{L} \nabla Z_{v}(x, f) \tilde{S}_{\dot{v} \dot{\varepsilon}}(x) n_{s}(x) \nabla Z_{\varepsilon}^{*}(x, f) d x \\
& +\int_{0}^{L} \nabla Z_{\varepsilon}(x, f) \tilde{S}_{\dot{\varepsilon} \dot{v}}(x) n_{s}(x) \nabla Z_{v}^{*}(x, f) d x
\end{aligned}
$$

We can see on Figure 7 that the main contribution to the total noise comes from the first term of the sum associated with velocity and that the contribution of sum of the two last terms is negative and practically negligible.

\section{CONCLUS:ON}

Using the SPM, we have calculated at the same microscopic level the noise sources associated with velocity and energy accelerations and the generalised impedance fields. These sources are by definition independent of the frequency and uncorrelated in time and space; they are obtained using the collision matrix of the evolution operator. The small signal generalised impedance fields are calculated by studying the evolution in $\mathbf{k}, \mathbf{r}$ and $t$, of perturbations of the velocity or energy introduced on the local distribution function of the carriers. Using these two quantities the spectral density of voltage fluctuation is obtained easily by a spatial integration.

\section{Acknowledgements}

This work have been performed with the support of the high-level grant DRB4/MDL/no 99-30 of the French Ministère de 1'Education Nationale, de la Recherche et de 1'Industrie and the FrenchLithuanian bilateral Cooperation no 5380 of CNRS.

\section{References}

[1] Shockley, W., Copeland, J. A. and James, R. P., "Quantum Theory of atoms, molecules and the Solid State", Ed. P.O. Lowdin, Academic, New York, 1966.

[2] Nougier, J. P., Vaissiere, J. C. and Gontrand, C. (1983). "Two-poing correlation of diffusion noise sources of hot carriers in semiconductors", Phys. Rev. Lett., p. 513.

[3] Shiktorov, P., Starikov, E., Gruzhinskis, V., Reggiani, L., Gonzalez, T., Mateos, J., Pardo, D. and Varani, L. (1998). "Acceleration fluctuation scheme for diffusion noise source within a generalised impedance field method", Phys. Rev. $B$, p. 11866 .

[4] Vaissière, J. C., Nougier, J. P., Varani, L., Houlet, P., Hlou, L., Reggiani, L., Starikov, E. and Shiktorov, P., "Numerical solution of the pertubed Boltzmann equation in frequency and time domains", 3rd IWCE, (Portland, 1994), pp. 53-56, Ed. Goodnick, M., Oregon State University.

[5] Aboubacar, M., Houlet, P., Nougier, J. P., Varani, L. and Vaissière, J. C. (1996). "Transport and noise calculations in semiconductor structures with the scattered packet method", Lithuanian Journal of Physics, 36(6), 583-587.

[6] Vaissière, J. C. (1997). "The scattered packet method as a new tool for microscopic investigation of noise", 14th $I C N F$, Leuven, Belgium, Eds. Claeys, C. and Simoen, E. (World Scientific), pp. 130-135.

[7] Shiktorov, P., Starikov, E., Gruzhinskis, V., Gonzalez, T., Mateos, J., Pardo, D., Reggiani, L., Varani, L. and Vaissière, J. C., "Langevin Forces and Generalized Transfer Fields for Noise Modeling in Deep Submicron Devices", 7th IWCE, (Glasgow, 2000).

[8] Gaubert, P., "Nouvelle méthode de simulation du bruit électronique dans les composants submicroniques", Phd report (Montpellier, December, 1999). 

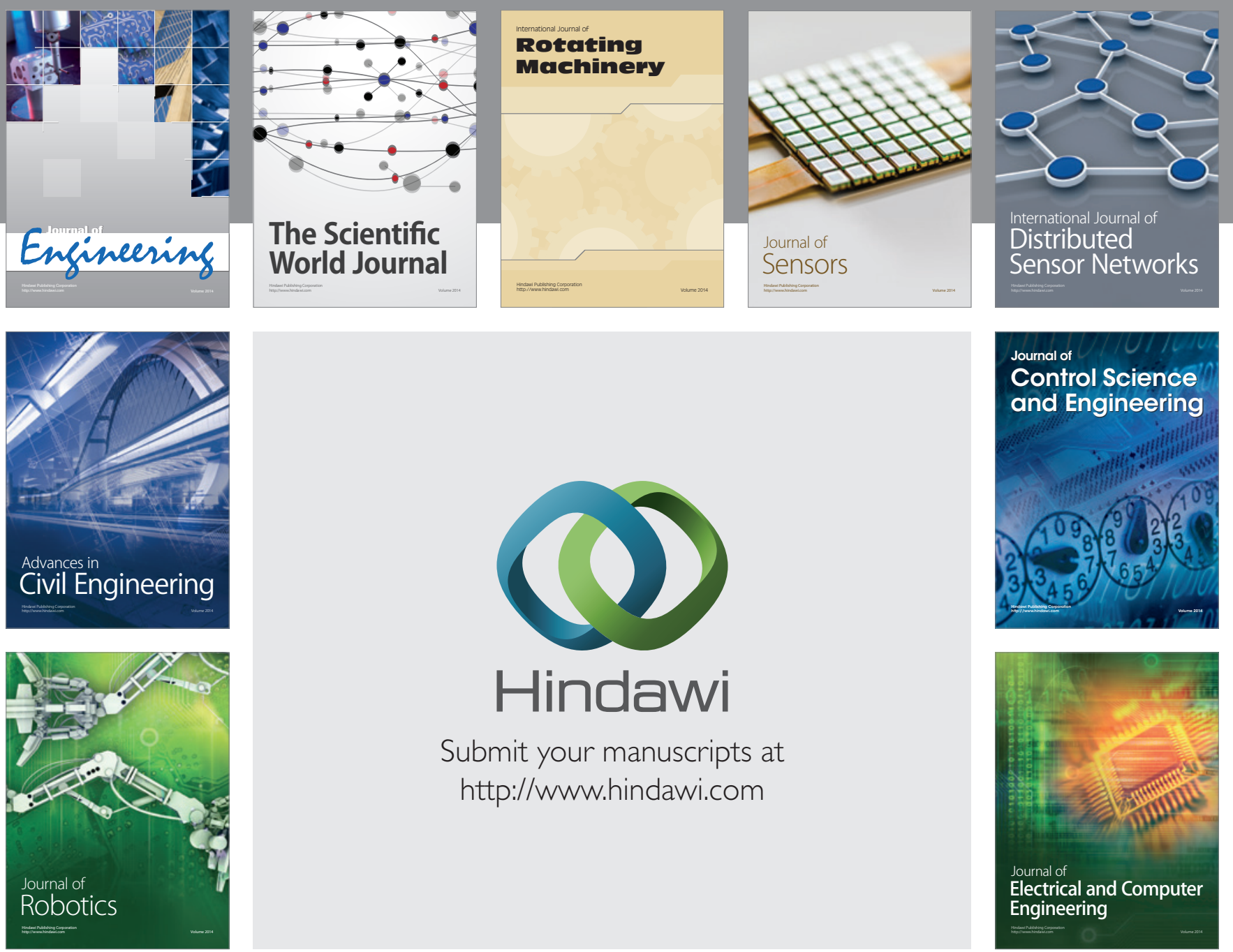

Submit your manuscripts at

http://www.hindawi.com
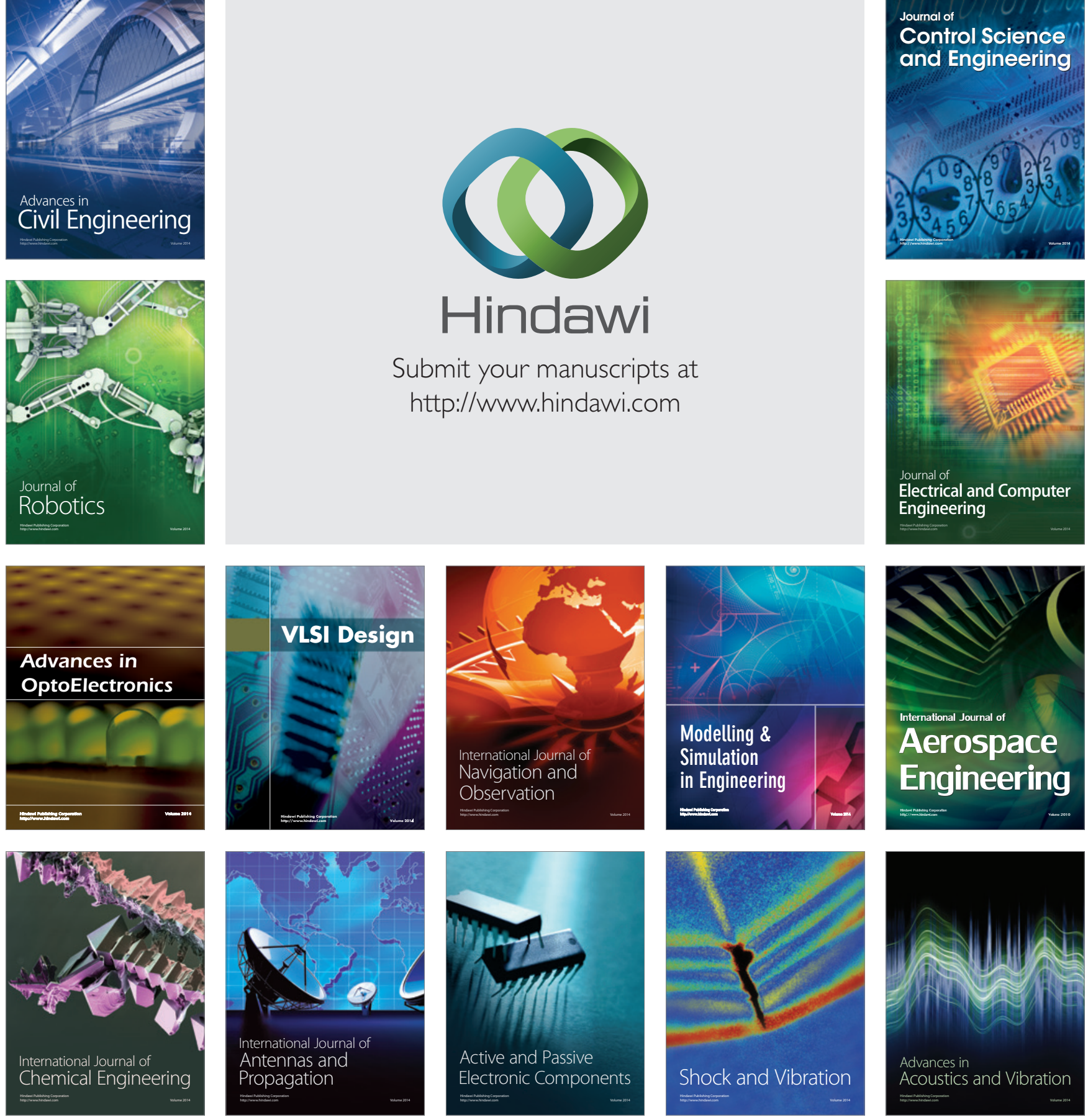